\title{
A rat lever designed to minimize partial presses*
}

\author{
RICHARD F. SMITH \\ University of Utah, Salt Lake City, Utah 84112 \\ and

\section{GARY A. FLINT \\ Mendocino State Hospital, Mendocino, California 95460}

The force required to move commercial spring-loaded rat levers increases with lever displacement. This characteristic allows the rat to terminate barpresses before they are recorded. Such partial presses are unlikely to occur when the force requirement rapidly decreases as displacement increases. This inverse relationship between force and displacement can be achieved by requiring the rat to lift the tail of the bar out of a magnetic field; the consequence is that the bar "falls out from under" the $S$, and nearly all movements of the bar result in switch closure. The use of electromagnetic fields provides two additional advantages: the force requirement can be established and monitored remotely.

The barpress in operant experiments is usually defined in terms of an electrical signal provided by the closure of a microswitch attached to the response lever. Since the lever can be moved easily without closing the microswitch, most Es consider it necessary to provide their rats with a feedback signal at switch closure in order to minimize response variability and improve performance. In typical operant studies, the data needec for such a demonstration are unavailable because movements of the bar that fail to produce feedback are also unrecorded. Further, it is difficult to assess the extent to which auditory or visual feedback signals may influence the effects of other discriminative stimuli present in the situation, because the feedback signals are confounded with the dependent variable (barpressing).

The E may wish to provide feedback directly associated with moving the lever and minimally associated with the visual and/or auditory stimuli used as independent variables. A reasonable choice would be proprioceptive feedback produced when the lever is moved. This would require a lever which moved the same distance on each press and always produced switch closure. This situation cannot be obtained with commercial levers that either require the rat to overcome a force provided by an adjustable spring or require the rat to overcome some combination of a weight attached to the opposite end (tail) of the lever and a spring contained in the microswitch. In the first case, the force

*This work was supported by NIMH Research Grant MH 10474 and PHS Research Grant HD 05026. The author wish to thank John Waltke and Eugene Izatt for their technical astistance. Reprints may be obtained from R. F. Smith, Department of Psychology, University of Utah, Salt Lake City, Utah 84112 . requirement increases as displacement increases and the rat can easily stop pressing at any time prior to switch closure. In the second case, the force requirement is relatively constant until the microswitch spring is encountered, then the force increases linearly as in the first case. In both cases, the lever moves more easily at the point of initial displacement than it does at switch closure and the rat can terminate the press before it is recorded.

Anger (1956) developed a rat lever with force requirement provided by a combination of a permanent magnetic field and a weight. His bar required $15 \mathrm{~g}$ for initial displacement and $8 \mathrm{~g}$ for switch closure. The decrease in required force at switch closure results from the decrease in the influence of the magnetic field when the bar is pressed. If one arranged a balanced bar opposed only by a magnetic field, the bar would "fall out from under" the $S$ once a force necessary for initial displacement of the bar was obtained. That is, the force required to move the bar would be inversely proportional to the square of the distance from the source of the magnetic field. Such a situation should greatly reduce the frequency of unrecorded partial presses, and movement feedback would be correlated only with effective (recorded) presses.

The use of electromagnetic fields results in two additional advantages: (a) the force requirement can be monitored remotely by measurement of the voltage drop across the coil of the electromagnet, and (b) the force requirement can be varied by changing this voltage as a consequence of either the S's or the E's behavior. The more intriguing case of $S$ control over bar force can be implemented quite easily: investigators using relay equipment can establish response-force contingencies by allowing barpresses to move a stepper through a pattern of fixed resistors wired in series with the electromagnet coil. Time-force contingencies can be programmed by arranging clock control over the stepper. Response rate (titration) contingencies can be arranged by combining techniques with the aid of a bidirectional stepper (i.e., time moves the stepper in one direction, while responses move the stepper in the opposite direction). Users of solid-state modular equipment or laboratory computers can deal with the problem in a similar fashion or employ digital-to-analog converters available through their equipment supplier. The supplier should be able to provide the interfacing details for conversion of voltages from his device to levels necessary to produce the desired force range on the bar.

The authors have built and tested some 15 bars with force requirements determined by the strength of magnetic fields. Some of the bars have been in routine use for 5 years and have continued to function reliably. 


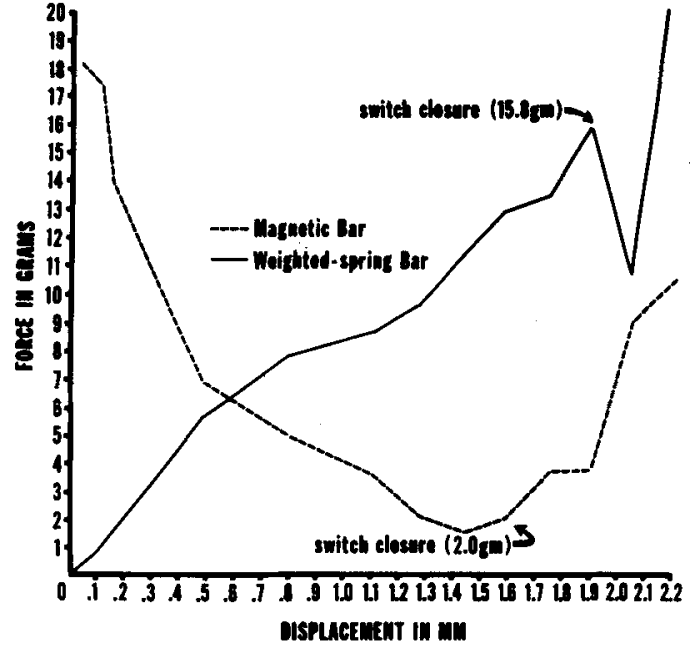

Fig. 1. The relationship between force and displacement for a commercial weighted-spring bar (solid line) and the magnetic bar (dotted line).

One of these veterans was chosen to provide the weight vs displacement data plotted in Fig. 1. The magnetic field for this bar was provided by an electromagnet (Potter and Brumfield 5KAA8 coil with a BOLL core). The data plotted in Fig. 1 were obtained with a coil voltage of $8.3 \mathrm{~V} \mathrm{dc}$. A force vs displacement function based on data obtained from a commercial lever is also shown in the figure. The force requirement for the commercial bar was produced by a combination of weight and spring. A stack of washers attached to the tail of the bar acted as the weight; a microswitch used to record responses contained the spring. The force vs displacement measurements were obtained by displacing the bars a known distance with a modified micrometer and then determining the force necessary to produce additional movement of the bar. The force necessary for additional movement was produced and measured with a spring scale attached to the lever. The force on the scale was recorded when downward displacement of the scale resulted in a loss of electrical contact between the lever and the shaft of the micrometer.

The magnetic bar began to move with an initial displacement force of $18.3 \mathrm{~g}$, and switch closure occurred with a force of $2.0 \mathrm{~g}$. Displacement to closure was $1.59 \mathrm{~mm}$. The weighted-spring bar began to move with a force of less than $1 \mathrm{~g}$, and switch closure occurred with a force of $15.8 \mathrm{~g}$. Displacement to closure was $1.90 \mathrm{~mm}$.

In general, the force requirement for the commercial bar increased linearly with displacement and revealed the effect of the microswitch spring. Since the switch lever was designed to contact the tail of the bar when the bar was in its resting position, both sources of opposition to the press (weight and spring) exerted their influence from initial displacement to switch closure.

A very different function was plotted for the magnetic bar. In general, the function was negatively accelerated and revealed the effect of moving the tail of the bar out of the electromagnetic field. The slight increase in the force requirement just prior to switch closure was produced by the action of the microswitch spring. The force requirement for both bars increased abruptly after switch closure. The increase resulted primarily from the microswitch springs and the displacement end-stops.

Two unsuccessful attempts were made to demonstrate behaviorally the superiority of the magnetic bar. One attempt involved the use of a drinkometer circuit arranged to signal the number of times the bars left their resting position. Completed responses were recorded in the usual manner, and the difference between drinkometer circuit counts and completed responses was taken as a measure of partial pressing. While the difference was much greater for Ss run with the commercial bar (as high as $48 \%$ partial presses), Ss run with the magnetic bar occasionally produced a negative percentage of partial presses (i.e., completed responses exceeded drinkometer counts because the rats failed to allow the bar to return to its resting position). These might be considered "inverted" partial presses, and, because such behavior was also possible with the commercial bar, it was concluded that we had developed a faulty electrical definition of partial pressing.

The second attempt involved the use of independent human Os to record partial pressing while rats operated the bars for reinforcement under continuous and fixed-ratio schedules. The $O$ data was judged unreliable because of lack of agreement on the definition of partial presses and reported differences in response topography between the two bars. The general, and perhaps biased, opinion of the Os was that behavior on the magnetic bar was more stereotyped and resulted in fewer partial presses than did behavior on the commercial bar. Maintenance of contact with both bars suggested that "inverted" partial pressing occurred in both cases.

\section{CONSTRUCTION}

The basic frame of the bar mechanism is made of $11 / 2 \times 2$ in. stock channel aluminum milled to a height of $1 \frac{1}{4}$ in. The dimensions are given in Fig. 2. Hole placement for adjustment screws is not critical, but can be estimated from the drawing. The bar consists of 14-ga magnetic stainless steel bent and silver soldered to give a $1 / 2 \times 1-5 / 16$ in. operandum to be inserted $5 / 8$ in. into the aluminum channel. The bearings for the bar consist of 1/2-in. 8/32-hex socket set screws milled and polished to give a conical point on the end. The conical ends are threaded against the bar, which has conical receptacles made by partially drilling the bar at the appropriate locations. The tail of the bar extends over a relay magnet. By varying the voltage across the coil, the force required to deflect the bar can be adjusted. The spacing between the core and the tail of the bar is adjustable either by Screw B and/or by spacers under the core. This feature allows adjustment of the effort required to hold 
Fig. 2. Front-, side-, and rear-view construction diagrams of the magnetic bar. Measurements are given in inches. Measurements not included may be estimated from the figure.
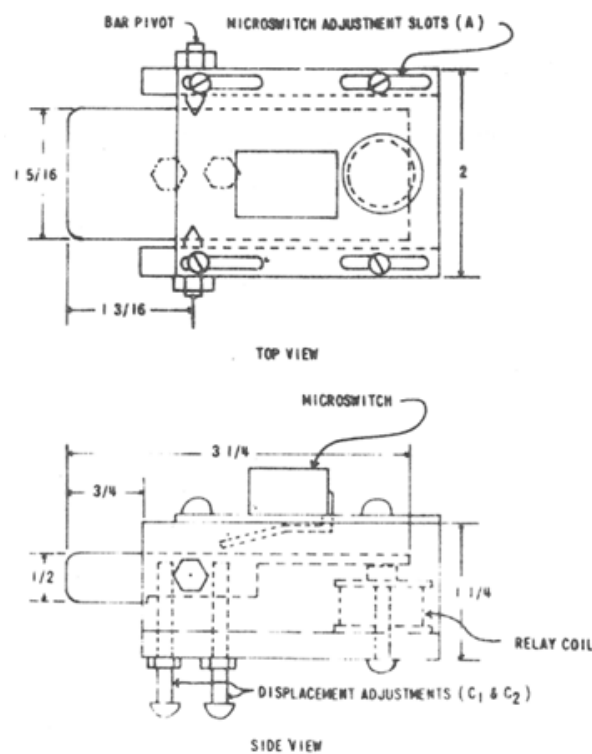

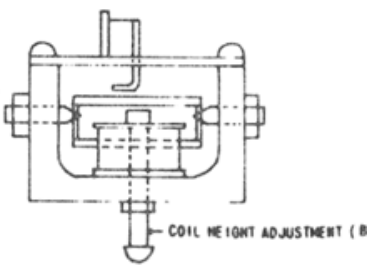

aear vien the bar in the down position for a given force required to deflect the bar. A snap-action microswitch was used in the bar described here, although other types of switches can be adapted for use with this design. For example, the standard 5-A relay contacts may be preferred in some applications. The switch mounting place is adjustable (A) to permit operation of the switch at any point in the arc of bar movement. The limits of displacement are adjustable with Screws $C_{1}$ and $C_{2}$.

Waltke Scientific Company (630 E. University St., Bloomington, Indiana 47401) has agreed to manufacture these bars at $\$ 49.95$ each, Rectractable magnetic bars are quoted at $\$ 54.95$, and bars designed to users' specifications can be negotiated.

\section{REFERENCE}

Anger, $D$. The dependence of interresponse times upon the relative reinforcement of different interresponse times. Journal of Experimental Psychology, 1956, 53, 145-161.

(Received for publication July 10, 1972; accepted August 18, 1972.) 